\title{
Annihilation of the electron-positron pairs in polyelectrons
}

\author{
Alexei M. Frolov and Farrukh A. Chishtie \\ Department of Applied Mathematics, University of Western Ontario, London, Ontario, Canada N6A 5Br
}

February 20, 2019

\begin{abstract}
Annihilation of the electron-positron pairs (or $\left(e^{-}, e^{+}\right)$-pairs, for short) in various polyelectrons $e_{n}^{+} e_{m}^{-}=e_{m}^{-} e_{n}^{+}$ (where $n \geq 1$ and $m \geq 1$ ) is considered. In particular, we discuss the three- and four-photon annihilation of the $\left(e^{-}, e^{+}\right)$-pairs in the three-body $\mathrm{Ps}^{-}$ion and four-body bi-positronium system $\mathrm{Ps}_{2}$. It is shown that the five-body $e_{2}^{+} e_{3}^{-}$ion is an unbound system. The closed form expression is derived for the amplitude-square $|M|^{2}$ of the threephoton annihilation of $\left(e^{-}, e^{+}\right)$-pair at arbitrary energies of the colliding particles. Analogous amplitude-square $|M|^{2}$ for the four-photon annihilation is reduced to the form which convenient for future analytical calculations. A method which can be used to produce macroscopic polyelectrons is briefly discussed.
\end{abstract}

PACS: 36.10.-k and 36.10.Dr 


\section{Introduction}

Annihilation of the electron-positron pairs (or $\left(e^{-}, e^{+}\right)$-pairs, for short) in various polyelectrons $e_{n}^{+} e_{m}^{-}$is considered. The polyelectrons discussed in this work include the three-body positronium ion $\mathrm{Ps}^{-}\left(e^{+} e_{2}^{-}\right)$and four-body bipositronium system $\mathrm{Ps}_{2}\left(e_{2}^{+} e_{2}^{-}\right)$. We also consider annihilation of the $\left(e^{-}, e^{+}\right)$-pairs in macroscopic polyelectrons, i.e. in the $e_{n}^{+} e_{m}^{-}$systems, where $n \approx N_{A}$ and $m \approx N_{A}$ and $N_{A} \approx 6.022 \cdot 10^{23}$ is the Avogadro number. Here and everywhere below in this work the notation $e^{+}$stands for the positron, while the notation $e^{-}$means the electron. Theory of polyelectrons and analysis of annihilation in such systems are required in many applications. Note that in small polyelectrons $e_{n}^{+} e_{m}^{-}$, where $n \leq 5$ and $m \leq 5$, the leading annihilation process is the annihilation of electronpositron pairs from bound states. In higher polyelectrons annihilation of the $\left(e^{-}, e^{+}\right)$-pairs from unbound states also contribute.

The positronium ion, bi-positronium and higher polyelectrons are of great interest in some applications to astrophysics [1], solid state physics 2] and other problems [3] - [5]. Most of such applications are related to the electron-positron pair annihilation in these polyelectrons. For instance, a long-standing, unsolved problem in astrophysics is to explain the formation and nature of an unknown and very intense source of positrons located in the center of our Galaxy. In fact, this source is located at a distance of $\approx 8 \mathrm{kpc}\left(1 \mathrm{kpc} \approx 3.086 \cdot 10^{16} \mathrm{~km}\right)$ from our Sun in the direction of Galactic center. It has spatial radius $\approx 1 k p c[1,[6$ and generates extremely large number of positrons per second $\left(\approx 1.3 \cdot 10^{43}\right.$ positrons 6 ] $)$. The emitted positrons later annihilate in that area which is usually called by the Galactic bulge 6. An intense emission of the $511 \mathrm{KeV}$ annihilation $\gamma$-quanta from Galaxy bulge indicates that the $\left(e^{-}, e^{+}\right)$-pair annihilation proceeds mainly from the bound states of various polyelectrons and positron compounds with some light atoms. Therefore, in order to make quantitative evaluations for the mentioned positron source one needs to estimate the relative probabilities of different annihilation processes in the $\mathrm{Ps}^{-}$ion, bi-positronium $\mathrm{Ps}_{2}$ and other polyelectrons. Note also that the macroscopic polyelectrons as well as the electron-positron plasma are the examples of the systems from different, non-Born-Oppenheimer world. Indeed, the masses of positive and negative particles is such systems are equal to each other. It is clear that the properties of non-Born-Oppenheimer systems differ quite substantially from the properties of regular atomic-molecular systems. This explains interest to polyelectrons from statistical physics. Another interesting application of polyelectrons is related to the energy production purposes. Indeed, it is easy to evaluate that the total annihilation of one gram of the electron-positron (1:1) mixture produces the energy $\approx 4.93 \cdot 10^{10} \mathrm{~J}$. The same amount of energy can be obtained, e.g., from thermal explosion of 11.8 tonnes of TNT. Briefly, the amount of energy released during complete annihilation of one gram of the electron-positron (1:1) mixture is quite comparable with the thermal energy released from the fission of one gram of $\mathrm{Pu}-239$ (16.4 tonnes TNT per gram) and from thermonuclear burning of one gram of the 1:1 deuterium-tritium mixture (13.8 tonnes TNT per gram). Note that the $\left(e^{-}, e^{+}\right)$-pair annihilation does not require any minimal critical density and/or threshold temperature for its ignition. On the other hand, the rate of energy release in any system undergoing annihilation rapidly increases at high compressions. This is also true for all working fusion/fission systems. At high densities the photons emitted during $\left(e^{-}, e^{+}\right)$-pair annihilation have significantly better probabilities to re-deposit their energy into surrounding atoms and electrons. A possibility to use the $\left(e^{-}, e^{+}\right)$-pair annihilation in highly compressed macroscopic polyelectrons seems to be very interesting for energy production purposes.

On the other hand, the annihilation of the $\left(e^{-}, e^{+}\right)$-pairs in various polyelectrons is an important problem of quantum electrodynamics. In general, such an annihilation proceeds with the emission of the two-, three-, ..., $n$-photons. The one- and zero-photon annihilations are also possible for many polyelectrons (see below). Accurate evaluation of the corresponding annihilation rates is extremely complicated QED problem. All mentioned problems attract a very significant attention to polyelectrons. Note that the existence of the bound $\mathrm{Ps}^{-}$ion has been predicted long ago by Ruark [7] and Wheeler [8]. Rigorously, the boundness of the ground state in the $\mathrm{Ps}^{-}$ion was shown by Hylleraas [9]. Hylleraas and Ore were also shown the boundness of the bi-positronium $\mathrm{Ps}_{2}$. In his work [8] Wheeler also discussed [8] a possibility to create some higher polyelectrons $e_{n}^{+} e_{m}^{-}$, where $n \geq 2$ and $m \geq 2$. Below, our main goal is to consider the annihilation of the $\left(e^{-}, e^{+}\right)$-pairs in various polyelectrons. By analyzing $\left(e^{-}, e^{+}\right)$-pair annihilation in the ground states of the $\mathrm{Ps}^{-}$ion and bi-positronium $\mathrm{Ps}_{2}$ we want to bring attention to a number of problems which still remain unsolved. In particular, we derive the explicit and closed expression for the $|M|^{2}$ factor for the three-photon annihilation of the $\left(e^{-}, e^{+}\right)$-pair at arbitrary energies of the colliding electron and positron. The analogous expression for the amplitude-square $|M|^{2}$ in the case of four-photon annihilation has been reduced to the form which is convenient for future considerations. Another goal of this study is to discuss the new approach which can be used to create macroscopic polyelectrons $e_{n}^{+} e_{m}^{-}$(or polyleptons [11]), where $n \approx N_{A}$ and $m \approx N_{A}$, where $N_{A}$ is the Avogadro number.

This work has the following structure. In the next section we briefly review the annihilation results known for the $\mathrm{Ps}^{-}$ion and bi-positronium $\mathrm{Ps}_{2}$. The three- and four-photon annihilation of the $\left(e^{-}, e^{+}\right)$-pair is considered in 
the third and fourth sections, respectively. Here we also discuss the stability of the $e_{2}^{+} e_{3}^{-}$ion and tri-positronium $\mathrm{PS}_{3}\left(=e_{3}^{+} e_{3}^{-}\right)$. The creation of macroscopic polyelectrons is discussed in the fifth section. Concluding remarks can be found in the last section.

\section{Positron annihilation in the positronium ion}

Let us discuss the $\left(e^{-}, e^{+}\right)$-pair annihilation in the three-body positronium ion $\mathrm{Ps}^{-}\left(e^{+} e_{2}^{-}\right)$. Annihilations of the $\left(e^{-}, e^{+}\right)$-pair in the three-body $\mathrm{Ps}^{-}$ion may proceed with the emission of the two and three photons, respectively. The four-, five- and more photon annihilations also occur in the $\mathrm{Ps}^{-}$ion, but the probabilities of such processes are much smaller (about four-photon annihilation, see below in Section IV). In addition to these many-photon processes the one-photon annihilation [12] is possible in the $\mathrm{Ps}^{-}$ion. The formula for one-photon annihilation rate $\Gamma_{1 \gamma}$ in the $\mathrm{Ps}^{-}$ion takes the form [12, 13 .

$$
\Gamma_{1 \gamma}=\frac{64 \pi^{2}}{27} \cdot \alpha^{8} \cdot c \cdot a_{0}^{-1} \cdot\left\langle\delta_{321}\right\rangle=1065.7569198 \cdot\left\langle\delta_{321}\right\rangle \sec ^{-1}
$$

where $\alpha=0.7297352568 \cdot 10^{-2}$ is the fine structure constant, $c=0.299792458 \cdot 10^{9} \mathrm{~m} \cdot \mathrm{sec}^{-1}$ is the speed of light in vacuum and $a_{0}$ is the Bohr radius which equals $0.5291772108 \cdot 10^{-10} \mathrm{~m}$. In this study the values of all physical constants are taken from [14. By using the expectation value of the triple delta-function $\left\langle\delta_{321}\right\rangle \approx 3.58891735 \cdot 10^{-5}$ from our most recent highly accurate computations [15] one finds that $\Gamma_{1 \gamma} \approx 3.82491 \cdot 10^{-2} \mathrm{sec}^{-1}$. Note that the total non-relativistic energy obtained with the same wave functions [15] is $E_{n r}=-0.2620050702329801077703745$ a.u., i.e. the most accurate value to-date. Now, consider the two- and three-photon annihilations of the positronium ion $\mathrm{Ps}^{-}$. It can be shown that the corresponding annihilation rates are uniformly related to the analogous values determined for the singlet/triplet bound states of the electron-positron pair (= positronium $\operatorname{Ps}\left(e^{+} e^{-}\right)$, for short). In general, the $\left(e^{-}, e^{+}\right)$-pair can be either in the singlet state, or in the triplet state. Annihilation of the singlet $\left(e^{-}, e^{+}\right)$- pair can proceed with the emission of the even number of photons (two, four, six, etc). In contrast with this, annihilation of the triplet $\left(e^{-}, e^{+}\right)$-pair produces only odd number of photons (three, five, etc). The leading twoand three-photon annihilations of the electron-positron pairs are of great interest in applications. The corresponding annihilation rates $\Gamma_{2 \gamma}$ and $\Gamma_{3 \gamma}$ for the bound singlet/triplet $\left(e^{-}, e^{+}\right)$-pair can be written in the following forms [16]

$$
\Gamma_{2 \gamma}=4 \pi \alpha^{4} c a_{0}^{-1}\left[1-\frac{\alpha}{\pi}\left(5-\frac{\pi^{2}}{4}\right)\right]\left\langle\delta_{+-}\right\rangle \approx 4 \cdot 50.17280269804 \cdot 10^{9}\left\langle\delta_{+-}\right\rangle \sec ^{-1}
$$

where $\delta_{+-}$is the electron-positron delta-function, and

$$
\Gamma_{3 \gamma}=\frac{16\left(\pi^{2}-9\right)}{9} \alpha^{5} c a_{0}^{-1}\left\langle\delta_{+-}\right\rangle \approx \frac{4}{3} \cdot 1.35927229774 \cdot 10^{8}\left\langle\delta_{+-}\right\rangle \sec ^{-1}
$$

respectively. Note that each of these two formulas explicitly contain the expectation value of the electron-positron delta-function $\delta_{+-}$. The expression for the two-photon annihilation rate $\Gamma_{2 \gamma}$, Eq.(2), also includes the lowest order radiative correction to the two-photon annihilation rate [17].In applications to the polyelectron systems $e_{n}^{+} e_{m}^{-}$the formulas, Eq.(2) and Eq.(3), must be multiplied by the total number of the singlet/triplet electron-positron pairs $(N)$ and corresponding statistical weights of the considered singlet/triplet spin states. In particular, for the $\mathrm{Ps}^{-}$ion we have $m=2$ and $N=2$, while the statistical weights of the singlet and triplet states equal $\frac{1}{4}$ and $\frac{3}{4}$, respectively. Therefore, from the formulas presented above one finds

$$
\Gamma_{2 \gamma}=N \pi \alpha^{4} c a_{0}^{-1}\left[1-\frac{\alpha}{\pi}\left(5-\frac{\pi^{2}}{4}\right)\right]\left\langle\delta_{+-}\right\rangle \approx 100.3456053781 \cdot 10^{9}\left\langle\delta_{+-}\right\rangle \sec ^{-1}
$$

and

$$
\Gamma_{3 \gamma}=N \frac{4\left(\pi^{2}-9\right)}{3} \alpha^{5} c a_{0}^{-1}\left\langle\delta_{+-}\right\rangle \approx 2.718545954 \cdot 10^{8}\left\langle\delta_{+-}\right\rangle \sec ^{-1},
$$

The sum of the $\Gamma_{2 \gamma}$ and $\Gamma_{3 \gamma}$ annihilation rates for the $\mathrm{Ps}^{-}$ion is usually called the total annihilation rate $\Gamma$ [18], [19]. The explicit formula for the total annihilation rate $\Gamma$ takes the form

$$
\begin{aligned}
\Gamma & =\Gamma_{2 \gamma}+\Gamma_{3 \gamma}=N \pi \alpha^{4} \cdot c \cdot a_{0}^{-1} \cdot\left[1-\alpha \cdot\left(\frac{17}{\pi}-\frac{19 \pi}{12}\right)\right] \cdot\left\langle\delta_{+-}\right\rangle \\
& =100.61745997357 \cdot 10^{9} \cdot\left\langle\delta_{+-}\right\rangle \mathrm{sec}^{-1}
\end{aligned}
$$


By using the best-to-date expectation value for the electron-positron delta-function in the $\mathrm{Ps}^{-}$ion $[15]\left(\left\langle\delta_{+-}\right\rangle \approx\right.$ $2.0733198005180(15) \cdot 10^{-2}$ a.u.) one finds from the formulas given above $\Gamma_{2 \gamma} \approx 2.08048530525 \cdot 10^{9} \sec ^{-1}, \Gamma_{3 \gamma} \approx$ $5.6364151550 \cdot 10^{6} \mathrm{sec}^{-1}$ and $\Gamma \approx 2.0861217204 \cdot 10^{9} \mathrm{sec}^{-1}$. In the laboratory measurements of the total annihilation rate $\Gamma$ in the $\mathrm{Ps}^{-}$ion $\left[20\right.$ it was found that $\Gamma \approx 2.09 \cdot 10^{9} \mathrm{sec}^{-1}$.

\subsection{Positron annihilation in bi-positronium}

The bi-postronium $\mathrm{Ps}_{2}\left(\right.$ or $e_{2}^{+} e_{2}^{-}$) is the four-body system of two electrons and two positrons. The electron-positron annihilation in this four-body system has been discussed in our earlier work [21]. Briefly, all formulas for the corresponding annihilation rates from the previous section can also be used for bi-positronium $\mathrm{Ps}_{2}$. The only difference can be found in the total number of electron-positron pairs. In bi-positronium one finds $N=2 \cdot 2=4$, while in the $\mathrm{Ps}^{-}$ion $N=2$. In general, in the polyelectron which contains $m$ electrons and $n$ positrons we have $N=m \cdot n$. Note, however, that in addition to the processes considered above, in bi-positronium $\mathrm{Ps}_{2}$ the zero-photon annihilation of the $\left(e^{-}, e^{+}\right)$-pair is also possible. The corresponding annihilation rate is [22]

$$
\Gamma_{0 \gamma}=\frac{147 \sqrt{3} \pi^{3}}{2} \cdot \alpha^{12}\left(c a_{0}^{-1}\right) \cdot\left\langle\delta_{++--}\right\rangle=5.099189 \cdot 10^{-4} \cdot\left\langle\delta_{++--}\right\rangle \sec ^{-1}
$$

where $\left\langle\delta_{++--}\right\rangle$is the expectation value of the four-particle delta-function in bi-positronium $\mathrm{Ps}_{2}$. The one-photon annihilation in the $\mathrm{Ps}_{2}$ system may also proceed with the emission of either one fast positron, or one fast electron (in the $\mathrm{Ps}^{-}$ion only fast electron can be emitted during such a process). In respect to this, the total one-photon annihilation rate in bi-positronium $\mathrm{P}_{\mathrm{S}_{2}}$ is written in the form

$$
\Gamma_{1 \gamma}=\frac{128 \pi^{2}}{27} \cdot \alpha^{8}\left(c a_{0}^{-1}\right) \cdot\left\langle\delta_{+--}\right\rangle=2.1315138 \cdot 10^{4} \cdot\left\langle\delta_{+--}\right\rangle \sec ^{-1}
$$

where $\left\langle\delta_{+--}\right\rangle=\left\langle\delta_{++-}\right\rangle$in the $\mathrm{Ps}_{2}$ system. The one-photon annihilation is followed by the emission of one fast electron/positron. The Lorentz $\gamma$-factor of the fast electron/positron in the $\mathrm{Ps}_{2}$ system emitted during onephoton annihilation is always bounded between 1 and 2. In general, to evaluate the corresponding annihilation rates in bi-positronium $\mathrm{Ps}_{2}$ one needs to know the expectation values of all electron-positron delta-functions, i.e. the $\left\langle\delta_{+-}\right\rangle,\left\langle\delta_{+--}\right\rangle\left(=\left\langle\delta_{++-}\right\rangle\right)$and $\left\langle\delta_{++--}\right\rangle$expectation values. Our most recent expectation values obtained for these delta-functions in the $\mathrm{Ps}_{2}$ system are $\left\langle\delta_{+-}\right\rangle=2.21039 \cdot 10^{-2},\left\langle\delta_{+--}\right\rangle=\left\langle\delta_{++-}\right\rangle=9.1995 \cdot 10^{-5}$ and $\left\langle\delta_{++--}\right\rangle=$ $4.596 \cdot 10^{-6}$ (all values are in atomic units). The wave function used in these calculations corresponds to the total energy of the $\mathrm{Ps}_{2}$ system is $E \approx-0.5160037901 a . u$. This wave function is very accurate, since the ground state energy obtained with this wave function is very close to the lower value produced in 23. By using these numerical values and formulas presented above one can determine the $\Gamma_{0}, \Gamma_{1}, \Gamma_{2}, \Gamma_{3}$ and $\Gamma$ annihilation rates for bi-positronium.

\subsection{Annihilation in higher polyelectrons}

Currently, the $\mathrm{Ps}^{-}$ion and bi-positronium $\mathrm{Ps}_{2}$ are the only two polyelectrons which have been extensively studied. The bound state computations of higher polyelectrons are significantly more difficult to perform. In fact, the boundness of higher polyelectrons, including the five-body ion $e_{2}^{+} e_{3}^{-}$and six-body tri-positronium system $e_{3}^{+} e_{3}^{-}$is still an open question. Main problem here is related to very complex permutation symmetry of the wave functions. In general, the three electrons/positrons can form the six following spin states: the two doublet states with the total spin $S=\frac{1}{2}$ and four quartet states with $S=\frac{3}{2}$. It can be shown that in bound state computations of the $e_{2}^{+} e_{3}^{-}$and $e_{3}^{+} e_{3}^{-}$systems only doublet three-electron/positron states are important. Analogous spin states with the total spin $S=\frac{3}{2}$ do not contribute and can be ignored. In respect to this, below in this section we restrict ourselves to the consideration of the doublet three-electron/positron states only.

As follows from the results of our numerical study the bound ground state in the $e_{2}^{+} e_{3}^{-}$ion cannot be obtained, if only one (doublet) electron spin function $s_{1}=(\alpha \beta \alpha-\beta \alpha \alpha)$ used in computations. The computed energies converge to the lowest dissociation threshold for the $e_{2}^{+} e_{3}^{-}$ion $\mathrm{E}_{t r} \approx-0.5160037910$ a.u. which corresponds to the dissociation $e_{2}^{+} e_{3}^{-}=\mathrm{Ps}_{2}+e^{-}$. Analogous energies determined with the use of two electron spin functions $s_{1}=(\alpha \beta \alpha-\beta \alpha \alpha)$ and $s_{2}=(2 \alpha \alpha \beta-\beta \alpha \alpha-\alpha \beta \alpha)$ converge to the same value $\mathrm{E}_{t r}$, but in this case the actual convergence is much faster. In our present calculations of the $e_{2}^{+} e_{3}^{-}$ion we have used up to 400 radial basis functions (five-body gaussoids of ten relative coordinates) with carefully optimized non-linear parameters. The two electron spin functions $s_{1}$ and $s_{2}$ were also used. As follows from these computations the ground state in the $e_{2}^{+} e_{3}^{-}$ion was not bound, and therefore, very likely that the $e_{2}^{+} e_{3}^{-}$ion does not exist as a bound system. However, if one electron in the $e_{2}^{+} e_{3}^{-}$ion is completely 
separated from the core $\left(\mathrm{Ps}_{2}\right)$ and can be considered as a distinguishable particle, then the $e_{2}^{+} e_{3}^{-}$system is quite well bound. Its total energy in this case is $E \approx-0.555889$ a.u., while the threshold energy is the same as above $E \approx$ -0.5160037910 a.u. The expectation value of the electron-positron delta-function for the $e_{2}^{+} e_{3}^{-}$system is $\approx 0.0171795$ $\left(\Gamma_{2 \gamma} \approx 5.17114 \cdot 10^{9} \mathrm{sec}^{-1}\right)$. This means that the five-body ion $e_{2}^{+} e_{3}^{-}$can be considered as an asymptotically bound system and its structure is approximately represented as a motion of one electron in the field of bi-positronium $\mathrm{PS}_{2}$. The structure of the $e_{2}^{+} e_{3}^{-}$ion in this case correspond to the structure of highly excited Rydberg states in manyelectron atoms. Briefly, we can say that the five-body ion $e_{2}^{+} e_{3}^{-}$is an unbound system, but in some sense it can be represented as an 'asymptotically bound system'. The electron with small (zero) kinetic energy cannot move to the infinity from the central $\mathrm{Ps}_{2}$ system. In general, this also means that some resonances can be observed in the $e^{-}+$ $\mathrm{Ps}_{2}$ scattering at small energies. The situation with the tri-positronium system $\mathrm{Ps}_{3}=e_{3}^{+} e_{3}^{-}$is even more complicated. Our current computational results for this six-body system are converging to the energy which corresponds to the dissociation threshold for tri-positronium $\mathrm{Ps}_{3}=\mathrm{Ps}_{2}+\mathrm{Ps}(E \approx-0.7660037910$ a.u. $)$. Nevertheless, there is a chance that the tri-positronium is bound, since the neutral quasi-atom Ps produces some polarization of the bi-positronium system $\mathrm{Ps}_{2}$. In an ideal case, such a polarization is sufficient to form a bound state in the six-body system $\mathrm{Ps}_{2}+$ $\mathrm{Ps}=\mathrm{Ps}_{3}$, but it does not produce the destruction of the $\mathrm{Ps}_{2}$ system. Furthermore, in our computations we have used only one spin function for three positrons and two spin functions for three electrons. In actual computations all four independent spin functions must be used. Moreover, in the $\mathrm{P}_{3}$ system the overall contribution of the spin-spin interaction between electron and positron spin functions can be quite comparable with its total binding energy. A number of other factors may also contribute to the binding energy of tri-positronium. All such factors must be taken into account before the final conclusion about the stability of the $\mathrm{Ps}_{3}$ system is made. Currently, the boundness of the $\mathrm{Ps}_{3}$ system is an open question and its solution requires additional investigations.

It should be mentioned that in small polyelectrons $e_{n}^{+} e_{m}^{-}$annihilation of the $\left(e^{-}, e^{+}\right)$-pairs always proceeds from the bound (ground) state. However, if the total number of electron-positron pairs in polyelectrons increases, then their life-time against annihilation rapidly decreases $\tau \sim \frac{1}{m n}$. It follows from here that polyelectrons with $n \geq 50$ and $m \geq 50$ cannot be created in practice. Indeed, the life-time of such polyelectrons is shorter than $1 \cdot 10^{-13} \mathrm{sec}$, while the corresponding formation time certainly exceeds this value $\left(\approx 5 \cdot 10^{-13} \mathrm{sec}\right)$. This logic cannot be applied to linear polyelectrons, where each positron/electron has only finite number of surrounding antiparticles. In other words, various linear polyelectrons, including macroscopic polyelectrons, can be created in reality. This problem is discussed in the fifth section.

As mentioned above in higher polyelectrons annihilation of the $\left(e^{-}, e^{+}\right)$-pairs also proceeds from the states of unbound spectra. Therefore, it is important to consider annihilation from the states of unbound spectra in polyelectrons (= annihilation-in-flight). The overall rate of such a $n$-photon process is determined by the annihilation cross-section $\sigma_{n \gamma}$. The annihilation cross-section is the function of the energies of colliding particles. For instance, the total spin-averaged cross-section of the two-photon annihilation of the electron-positron pair written in the electron rest frame is given by the following expression [24]

$$
\sigma=\frac{\pi \alpha^{2}}{4 m^{2}} \frac{1-v^{2}}{v^{2}} \cdot\left[\left(3-v^{4}\right) \ln \left(\frac{1+v}{1-v}\right)-2 v\left(2-v^{2}\right)\right]
$$

where $v$ is the velocity of the colliding positron $e^{+}$. Here and in the two following sections we shall use the so-called relativistic units $c=1$ and $\hbar=1$. Note that in these units one finds $m=\alpha^{-1}$ and $a_{0}=\alpha^{-1}$ [25]. The last formula is reduced to the form

$$
\sigma=\frac{\pi \alpha^{2}}{2 m^{2}} \frac{1-v^{2}}{v} \cdot\left[\left(3-v^{4}\right) \sum_{k=1}^{\infty} \frac{v^{2 k-2}}{2 k-1}-2-v^{2}\right]
$$

As follows from this formula $\sigma \sim \frac{1}{v}$, if $v \rightarrow 0$. This can be expected, since the two colliding charged particles have opposite electric charges (electron $e^{-}$and positron $e^{+}$). Also, it is clear from the last formula that the product $\sigma v$ is not singular for small and zero positron velocities $v$.

\section{Three-photon annihilation}

The non-relativistic limit for the three-photon annihilation rate $\Gamma_{3 \gamma}$, Eq.(3), has been obtained almost 60 years ago [26] - 28]. The three-photon annihilation of the electron-positron pair is also considered in some modern papers (see, e.g., 31] and references therein). In many actual cases, however, it is also important to know the higher-order corrections to the annihilation rate obtained in the lowest-order approximation, Eq.(3). Briefly, one needs to obtain the formula for the $\Gamma_{3 \gamma}$ annihilation width which can be applied at arbitrary velocities of the colliding electron and positron. However, due to extreme complexity of the problem the general expression for the $\Gamma_{3 \gamma}$ annihilation 
rate has not been derived yet. In this study we want to derive the closed analytical formulas for the corresponding amplitude-square $|M|^{2}$. The principal conservation law in the case of three-photon annihilation of the $\left(e^{-}, e^{+}\right)-$pair is

$$
p_{1}+p_{2}=k_{1}+k_{2}+k_{3}
$$

where $p_{1}$ and $p_{2}$ are the 4 -vectors of electron and positron momenta, respectively. The $k_{1}, k_{2}, k_{3}$ are the 4 -vectors of photon momenta. Note that for the real particles we always have $p_{1}^{2}=m^{2}$ and $p_{2}^{2}=m^{2}$, while for the real photons $k_{i}^{2}=0(i=1,2,3)$. From Eq.(11) one finds

$$
m^{2}+p_{1} \cdot p_{2}=k_{1} \cdot k_{2}+k_{1} \cdot k_{3}+k_{2} \cdot k_{3}
$$

where $p_{1} \cdot p_{2}=E_{1} \cdot E_{2}-\mathbf{p}_{1} \cdot \mathbf{p}_{2}$ and $k_{i} \cdot k_{j}=\omega_{i} \cdot \omega_{j}-\mathbf{k}_{i} \cdot \mathbf{k}_{j}$. From Eq.(11) one can produce a number of other conservation laws, e.g.,

$$
m^{2}+p_{1} \cdot p_{2}=p_{1} \cdot k_{1}+p_{1} \cdot k_{2}+p_{1} \cdot k_{3}
$$

where $p_{1} \cdot k_{i}=E_{1} \cdot \omega_{i}-\mathbf{p}_{1} \cdot \mathbf{k}_{i}$ and

$$
k_{i} \cdot p_{1}+k_{i} \cdot p_{2}=k_{i} \cdot k_{j}+k_{i} \cdot k_{l}
$$

where $(i, j, l)=(1,2,3)$.

The Feynman diagram of the three-photon annihilation is shown on Fig. 1. The corresponding matrix element $M$ takes the form

$$
\begin{aligned}
M & =\bar{v}\left[\epsilon_{3} \frac{1}{p_{1}-k_{1}-k_{2}-m} \epsilon_{2} \frac{1}{p_{1}-k_{1}-m} \epsilon_{1}+\epsilon_{2} \frac{1}{p_{1}-k_{1}-k_{3}-m} \epsilon_{3} \frac{1}{p_{1}-k_{1}-m} \epsilon_{1}+\ldots\right. \\
& \left.+\epsilon_{i} \frac{1}{p_{1}-k_{j}-k_{l}-m} \epsilon_{j} \frac{1}{p_{1}-k_{l}-m} \epsilon_{l}+\ldots\right] u
\end{aligned}
$$

where $(i, j, l)=(1,2,3)$. The $v$ and $u$ are the positron and electron bi-spinors, respectively, while $k_{i}$ and $\epsilon_{i}(i=1,2$, $3)$ are the momentum and polarization of the $i$-th photon. The total number of terms in the amplitude $M$ equals six. Each of these six terms in Eq.(15) can be transformed in the following way [29] (e.g., for the first term)

$$
\begin{aligned}
M_{1}= & \bar{v}\left[\epsilon_{3} \frac{1}{p_{1}-k_{1}-k_{2}-m} \epsilon_{2} \frac{1}{p_{1}-k_{1}-m} \epsilon_{1}\right] u=A_{1} \bar{v}\left[\epsilon_{3}\left(p_{1}-k_{1}-k_{2}-m\right) \epsilon_{2} \times\right. \\
& \left.\left(p_{1}-k_{1}-m\right) \epsilon_{1}\right] u=A_{1} \bar{v}\left[\epsilon_{3}\left(p_{2}-k_{3}-m\right) \epsilon_{2}\left(p_{1}-k_{1}-m\right) \epsilon_{1}\right] u
\end{aligned}
$$

where

$$
A_{1}=\frac{1}{4\left(p_{1} \cdot k_{1}\right)\left(p_{2} \cdot k_{3}\right)}
$$

To simplify the expressions for the amplitudes $M_{1}$ and $M_{i}(i=2,3,4,5,6)$ we need to impose a few additional conditions on the photon polarization 4 -vectors $\epsilon_{i}(i=1,2,3)$. In particular, we shall assume that the following conditions are obeyed for the three $\epsilon_{i} 4$-vectors 30 ]

$$
\epsilon_{i} \cdot k_{i}=0 \quad, \quad \epsilon_{i} \cdot \epsilon_{i}=-1 \quad, \quad \epsilon_{i} \cdot p_{1}=0
$$

where $i=1,2,3$. It is to be noted that the last condition in Eq.(18) implies a redefinition of an arbitrary set of the photon polarization vectors, $\varepsilon_{i}^{\mu}=\epsilon_{i}^{\mu}-\frac{\epsilon_{i} \cdot p_{1}}{k_{i} \cdot p_{1}} \cdot k_{i}^{\mu}$ such that the incoming electron momenta, $p_{1}$ is orthogonal to all the polarization vectors. This retains the original normalization and transversality conditions (in the covariant Lorentz gauge) and leads to further simplification without any loss of generality in the calculation. For notational simplification, we choose $\epsilon_{i}$ over $\varepsilon_{i}$ for the rest of the paper. This simplification will also be applied in the four photon case. The five other conditions for the $p_{1}, p_{2}, k_{1}, k_{2}$ and $k_{3} 4$-vectors have been mentioned earlier $p_{1}^{2}=m^{2}, p_{2}^{2}=m^{2}$ 
and $k_{i}^{2}=0(i=1,2,3)$. By using these relations we can drastically simplify the expression for the amplitude Eq.(16). Indeed, by applying the relation $a b=2(a \cdot b)-b a$, where $a$ and $b$ are the two arbitrary 4-vectors, one finds

$$
\left(p_{1}-k_{1}+m\right) \epsilon_{1} u=-k_{1} \epsilon_{1} u+\epsilon_{1}\left(-p_{1}+m\right) u=-k_{1} \epsilon_{1} u
$$

since $\left(p_{1}-m\right) u=0$. Analogously, since $0=\bar{v}\left(p_{2}+m\right)$ we can simplify the remaining part of Eq.(16). Finally, we have

$$
M_{1}=-A \cdot \bar{v}\left[\epsilon_{3} k_{3} \epsilon_{2} k_{1} \epsilon_{1}\right] u+2 A\left(\epsilon_{3} \cdot p_{2}\right) \bar{v}\left[\epsilon_{2} k_{1} \epsilon_{1}\right] u
$$

where $A$ is given by the equation, Eq.(17), above. This expression can also be written in the following form

$$
M_{1}=M_{321}=A_{321} \cdot \bar{v}\left[2\left(\epsilon_{3} \cdot p_{2}\right) \epsilon_{2} k_{1} \epsilon_{1}-\epsilon_{3} k_{3} \epsilon_{2} k_{1} \epsilon_{1}\right] u
$$

where $A_{321} \equiv A_{1}$. The indexes in the last formula are uniformly related to the corresponding Feynman diagram (see Fig.1) by reading them from the right to the left. Analogously, for the $(i j l)$-diagram one finds

$$
M_{i j l}=A_{i j l} \cdot \bar{v}\left[2\left(\epsilon_{i} \cdot p_{2}\right) \epsilon_{j} k_{l} \epsilon_{l}-\epsilon_{i} k_{i} \epsilon_{j} k_{l} \epsilon_{l}\right] u
$$

where $(i, j, l)=(1,2,3)$ and

$$
A_{i j l}=\frac{1}{4\left(p_{1} \cdot k_{l}\right)\left(p_{2} \cdot k_{i}\right)}
$$

is the real value. The conjugate amplitude $M_{i j l}^{*}$ is

$$
M_{i j l}^{*}=A_{i j l} \cdot \bar{u}\left[2\left(\epsilon_{i} \cdot p_{2}\right) \epsilon_{l} k_{l} \epsilon_{j}-\epsilon_{l} k_{l} \epsilon_{j} k_{i} \epsilon_{i}\right] v
$$

The expression for the $|M|^{2}$ value is reduced to the sum of the six matrix element $M_{i j l}^{*} M_{321}$, where $(i, j, l)=$ $(1,2,3)$. The analytical formula for the $M_{i j l}^{*} M_{321}$ matrix element is

$$
M_{i j l}^{*} M_{321}=A_{i j l} A_{321} \cdot \bar{u}\left[2\left(\epsilon_{i} \cdot p_{2}\right) \epsilon_{l} k_{l} \epsilon_{j}-\epsilon_{l} k_{l} \epsilon_{j} k_{i} \epsilon_{i}\right] v \cdot \bar{v}\left[2\left(\epsilon_{3} \cdot p_{2}\right) \epsilon_{2} k_{1} \epsilon_{1}-\epsilon_{3} k_{3} \epsilon_{2} k_{1} \epsilon_{1}\right] u
$$

By averaging this expression over the initial spin of electron and positron states we find the expression

$$
\begin{aligned}
M_{i j l}^{*} M_{321}= & A_{i j l} A_{321} \cdot \operatorname{Tr}\left\{\left(\frac{p_{2}-m}{2 m}\right)\left[2\left(\epsilon_{i} \cdot p_{2}\right) \epsilon_{l} k_{l} \epsilon_{j}-\epsilon_{l} k_{l} \epsilon_{j} k_{i} \epsilon_{i}\right]\left(\frac{p_{1}+m}{2 m}\right) \times\right. \\
& {\left.\left[2\left(\epsilon_{3} \cdot p_{2}\right) \epsilon_{2} k_{1} \epsilon_{1}-\epsilon_{3} k_{3} \epsilon_{2} k_{1} \epsilon_{1}\right]\right\}=A_{i j l} A_{321} \cdot B_{i j l} }
\end{aligned}
$$

The trace $B_{i j l}$ can be written in the form

$$
B_{i j l}=\frac{1}{4 m^{2}}\left(B_{1}-m^{2} B_{2}\right)
$$

where

$$
\begin{aligned}
B_{1} & =\operatorname{Tr}\left\{\left[2\left(\epsilon_{i} \cdot p_{2}\right) p_{2} \epsilon_{l} k_{l} \epsilon_{j}-p_{2} \epsilon_{l} k_{l} \epsilon_{j} k_{i} \epsilon_{i}\right]\left[2\left(\epsilon_{3} \cdot p_{2}\right) p_{1} \epsilon_{2} k_{1} \epsilon_{1}-p_{1} \epsilon_{3} k_{3} \epsilon_{2} k_{1} \epsilon_{1}\right]\right\} \\
& =4\left(\epsilon_{i} \cdot p_{2}\right)\left(\epsilon_{3} \cdot p_{2}\right) \operatorname{Tr}\left[p_{2} \epsilon_{l} k_{l} \epsilon_{j} p_{1} \epsilon_{2} k_{1} \epsilon_{1}\right]-2\left(\epsilon_{i} \cdot p_{2}\right) \operatorname{Tr}\left[p_{2} \epsilon_{l} k_{l} \epsilon_{j} p_{1} \epsilon_{3} k_{3} \epsilon_{2} k_{1} \epsilon_{1}\right] \\
& -2\left(\epsilon_{3} \cdot p_{2}\right) \operatorname{Tr}\left[p_{2} \epsilon_{l} k_{l} \epsilon_{j} k_{i} \epsilon_{i} p_{1} \epsilon_{2} k_{1} \epsilon_{1}\right]+\operatorname{Tr}\left[p_{2} \epsilon_{l} k_{l} \epsilon_{j} k_{i} \epsilon_{i} p_{1} \epsilon_{3} k_{3} \epsilon_{2} k_{1} \epsilon_{1}\right] \\
& =4\left(\epsilon_{i} \cdot p_{2}\right)\left(\epsilon_{3} \cdot p_{2}\right) B_{1 a}-2\left(\epsilon_{i} \cdot p_{2}\right) B_{1 b}-2\left(\epsilon_{3} \cdot p_{2}\right) B_{1 c}+B_{1 d}
\end{aligned}
$$

and

$$
\begin{aligned}
B_{2} & =\operatorname{Tr}\left\{\left[2\left(\epsilon_{i} \cdot p_{2}\right) \epsilon_{l} k_{l} \epsilon_{j}-\epsilon_{l} k_{l} \epsilon_{j} k_{i} \epsilon_{i}\right]\left[2\left(\epsilon_{3} \cdot p_{2}\right) \epsilon_{2} k_{1} \epsilon_{1}-\epsilon_{3} k_{3} \epsilon_{2} k_{1} \epsilon_{1}\right]\right\} \\
& =4\left(\epsilon_{i} \cdot p_{2}\right)\left(\epsilon_{3} \cdot p_{2}\right) \operatorname{Tr}\left[\epsilon_{l} k_{l} \epsilon_{j} \epsilon_{2} k_{1} \epsilon_{1}\right]-2\left(\epsilon_{i} \cdot p_{2}\right) \operatorname{Tr}\left[\epsilon_{l} k_{l} \epsilon_{j} \epsilon_{3} k_{3} \epsilon_{2} k_{1} \epsilon_{1}\right] \\
& -2\left(\epsilon_{3} \cdot p_{2}\right) \operatorname{Tr}\left[\epsilon_{l} k_{l} \epsilon_{j} k_{i} \epsilon_{i} \epsilon_{2} k_{1} \epsilon_{1}\right]+\operatorname{Tr}\left[\epsilon_{l} k_{l} \epsilon_{j} k_{i} \epsilon_{i} \epsilon_{3} k_{3} \epsilon_{2} k_{1} \epsilon_{1}\right] \\
& =4\left(\epsilon_{i} \cdot p_{2}\right)\left(\epsilon_{3} \cdot p_{2}\right) B_{2 a}-2\left(\epsilon_{i} \cdot p_{2}\right) B_{2 b}-2\left(\epsilon_{3} \cdot p_{2}\right) B_{2 c}+B_{2 d}
\end{aligned}
$$


This means that we need to determine the eight following traces: the four traces which contain electron and positron momenta

$$
\begin{array}{r}
B_{1 a}=\operatorname{Tr}\left[p_{2} \epsilon_{l} k_{l} \epsilon_{j} p_{1} \epsilon_{2} k_{1} \epsilon_{1}\right] \\
B_{1 b}=\operatorname{Tr}\left[p_{2} \epsilon_{l} k_{l} \epsilon_{j} p_{1} \epsilon_{3} k_{3} \epsilon_{2} k_{1} \epsilon_{1}\right] \\
B_{1 c}=\operatorname{Tr}\left[p_{2} \epsilon_{l} k_{l} \epsilon_{j} k_{i} \epsilon_{i} p_{1} \epsilon_{2} k_{1} \epsilon_{1}\right] \\
B_{1 d}=\operatorname{Tr}\left[p_{2} \epsilon_{l} k_{l} \epsilon_{j} k_{i} \epsilon_{i} p_{1} \epsilon_{3} k_{3} \epsilon_{2} k_{1} \epsilon_{1}\right]
\end{array}
$$

and four traces which do not contain any electron and/or positron momenta

$$
\begin{aligned}
B_{2 a} & =\operatorname{Tr}\left[\epsilon_{l} k_{l} \epsilon_{j} \epsilon_{2} k_{1} \epsilon_{1}\right] \\
B_{2 b} & =\operatorname{Tr}\left[\epsilon_{l} k_{l} \epsilon_{j} \epsilon_{3} k_{3} \epsilon_{2} k_{1} \epsilon_{1}\right] \\
B_{2 c} & =\operatorname{Tr}\left[\epsilon_{l} k_{l} \epsilon_{j} k_{i} \epsilon_{i} \epsilon_{2} k_{1} \epsilon_{1}\right] \\
B_{2 d} & =\operatorname{Tr}\left[\epsilon_{l} k_{l} \epsilon_{j} k_{i} \epsilon_{i} \epsilon_{3} k_{3} \epsilon_{2} k_{1} \epsilon_{1}\right]
\end{aligned}
$$

The analytical expressions for these traces solve, in practice, the problem of three-photon annihilation for arbitrary energies of the colliding electron/positron. In fact, we have computed all these traces analytically. The explicit formulas for all individual traces, Eq.(32) - Eq.(39), as well as for $B_{i j l}$ can be found in 32.

The formulas given above correspond to the case when the polarizations of all photons (i.e. $\left.\epsilon_{i}, i=1,2,3\right)$ are known or can be easily measured. In many cases, however, the polarizations of the photons, i.e. the 4 -vectors $\epsilon_{i}(i$ $=1,2,3)$ cannot be determined. Therefore, in the formulas presented above one needs to compute the sums over all final photon polarizations.

We perform the polarization summation using the standard replacement $\sum_{\lambda=1,4} \epsilon_{\mu}^{(\lambda)} \epsilon_{\nu}^{(\lambda)}=-g_{\mu \nu}$. After performing the resulting traces, the expression $M_{i j l}^{*} M_{321}$, for the polarization summed case be compactly written as,

$$
M_{i j l}^{*} M_{321}=A_{i j l} \cdot A_{321} D_{i j l}
$$

for $(i, j, l)=(1,2,3)$ and $D_{i j l}$ denotes the resulting traces computed for each of these cases and given explicitly below as follows

$$
\begin{aligned}
D_{123} & =8\left[2\left(k_{1} \cdot k_{3}\right)^{2}+\left(k_{1} \cdot p_{2}\right)\left(k_{3} \cdot p_{1}\right)+\left(k_{1} \cdot p_{1}\right)\left(k_{3} \cdot p_{2}\right)+\left(k_{1} \cdot k_{3}\right)\left(2 m^{2}-p_{1} \cdot p_{2}\right)\right] \\
D_{132} & =8\left[\left(k_{1} \cdot k_{3}\right)\left(k_{2} \cdot p_{1}\right)-\left(k_{1} \cdot p_{2}\right)\left(4 k_{2} \cdot k_{3}+k_{2} \cdot p_{1}-2 k_{2} \cdot p_{2}\right)+\left(k_{1} \cdot p_{1}\right)\left(-k_{2} \cdot k_{3}\right.\right. \\
& \left.\left.+k_{2} \cdot p_{2}\right)+\left(k_{1} \cdot k_{2}\right)\left(k_{3} \cdot p_{1}\right)+\left(k_{1} \cdot k_{2}\right)\left(p_{1} \cdot p_{2}\right)\right] \\
D_{213} & =8\left[-\left(k_{1} \cdot k_{2}\right)\left(k_{3} \cdot p_{1}\right)+\left(k_{1} \cdot p_{2}\right)\left(k_{3} \cdot p_{1}\right)+\left(k_{1} \cdot p_{1}\right)\left(k_{2} \cdot k_{3}-k_{3} \cdot p_{2}\right)\right. \\
& \left.-4\left(k_{1} \cdot k_{2}\right)\left(k_{3} \cdot p_{2}\right)+2\left(k_{1} \cdot p_{2}\right)\left(k_{3} \cdot p_{2}\right)+\left(k_{1} \cdot k_{3}\right)\left(k_{2} \cdot p_{1}+p_{1} \cdot p_{2}\right)\right] \\
D_{231} & =\frac{16 k_{1} \cdot p_{2}}{m^{2}}\left[\left(k_{1} \cdot p_{1}\right)\left(-m^{2}-2 k_{2} \cdot k_{3}+k_{2} \cdot p_{2}+k_{3} \cdot p_{2}\right)-\left(k_{1} \cdot k_{2}+k_{1} \cdot k_{3}\right)\left(p_{1} \cdot p_{2}\right)\right. \\
& \left.+\left(k_{1} \cdot p_{2}\right)\left(k_{2} \cdot p_{1}+k_{3} \cdot p_{1}+2 p_{1} \cdot p_{2}\right)\right] \\
D_{312} & =\frac{16 k_{1} \cdot k_{2}}{m^{2}}\left[m^{4}+2 k_{3} \cdot p_{1}\left(m^{2}+k_{3} \cdot p_{2}\right)+2 m^{2}\left(p_{1} \cdot p_{2}\right)-\left(k_{3} \cdot p_{2}\right)\left(m^{2}+4 p_{1} \cdot p_{2}\right)\right] \\
D_{321} & =-\frac{32 k_{1} \cdot p_{2}}{m^{2}}\left[\left(k_{1} \cdot p_{2}\right)\left(k_{3} \cdot p_{1}\right)+\left(k_{1} \cdot p_{1}\right)\left(m^{2}-k_{3} \cdot p_{2}\right)-\left(k_{1} \cdot k_{3}\right)\left(k_{3} \cdot p_{1}+p_{1} \cdot p_{2}\right)\right]
\end{aligned}
$$

The total $|M|^{2}$ for the polarization summed case is therefore the sum of the six matrix element $M_{i j l}^{*} M_{321}$, where $(i, j, l)=(1,2,3)$.

\section{Four-photon annihilation rate}

The analysis of the four-photon annihilation is an important part of any annihilation analysis of polyelectrons. The reason for this is obvious and it follows from approximate evaluation of the $\Gamma_{4 \gamma}$ annihilation rate which indicates that $\Gamma_{4 \gamma} \approx \alpha^{2} \Gamma_{2 \gamma} \approx \frac{1}{6} \Gamma_{3 \gamma}$. In other words, the numerical value of $\Gamma_{4 \gamma}$ in any polyelectron is not negligible in comparison to the $\Gamma_{3 \gamma}$ annihilation rate. Therefore, the more accurate evaluation of the $\Gamma_{4 \gamma}$ annihilation rate must be performed in each of the polyelectrons, and in particular, for the $\mathrm{Ps}^{-}$ion and bi-positronium $\mathrm{Ps}_{2}$. The Feynman diagram which 
describes the four-photon annihilation of the electron-positron pair is shown on Fig.2. The principal 4-dimensional conservation law in this case is written in the form

$$
p_{1}+p_{2}=k_{1}+k_{2}+k_{3}+k_{4}
$$

where $p_{1}$ and $p_{2}$ are the 4 -vectors of electron and positron momenta, respectively. The $k_{1}, k_{2}, k_{3}, k_{4}$ are the 4 -vectors of photon momenta. From Eq.(47) one may derive a number of scalar conservation laws, e.g.,

$$
m^{2}+E_{1} \cdot E_{2}-\mathbf{p}_{1} \cdot \mathbf{p}_{2}=\sum_{i j(i<j)}\left(\omega_{i} \omega_{j}-\mathbf{k}_{i} \cdot \mathbf{k}_{j}\right)
$$

and

$$
\omega_{i} \cdot E_{1}-\mathbf{k}_{i} \cdot \mathbf{p}_{1}+\omega_{i} \cdot E_{2}-\mathbf{k}_{i} \cdot \mathbf{p}_{2}=\omega_{i}\left(\sum_{j(j \neq i)} \omega_{j}\right)-\mathbf{k}_{i} \cdot\left(\sum_{j(j \neq i)} \mathbf{k}_{j}\right)
$$

The matrix element for the four-photon annihilation rate takes the form

$$
\begin{aligned}
M= & \bar{v}\left[\epsilon_{4} \frac{1}{p_{1}-k_{1}-k_{2}-k_{3}-m} \epsilon_{3} \frac{1}{p_{1}-k_{1}-k_{2}-m} \epsilon_{2} \frac{1}{p_{1}-k_{1}-m} \epsilon_{1}+\right. \\
& \epsilon_{3} \frac{1}{p_{1}-k_{1}-k_{2}-k_{4}-m} \epsilon_{4} \frac{1}{p_{1}-k_{1}-k_{2}-m} \epsilon_{2} \frac{1}{p_{1}-k_{1}-m} \epsilon_{1}+\ldots \\
+ & \left.\epsilon_{i} \frac{1}{p_{1}-k_{j}-k_{l}-k_{n}-m} \epsilon_{j} \frac{1}{p_{1}-k_{l}-k_{n}-m} \epsilon_{l} \frac{1}{p_{1}-k_{n}-m} \epsilon_{n}+\ldots\right] u
\end{aligned}
$$

An arbitrary $(i j l n)$-term in this expression transforms in the following way

$$
\begin{aligned}
M_{i j l n} & =\bar{v}\left[\epsilon_{i} \frac{1}{p_{1}-k_{j}-k_{l}-k_{n}-m} \epsilon_{j} \frac{1}{p_{1}-k_{l}-k_{n}-m} \epsilon_{l} \frac{1}{p_{1}-k_{n}-m} \epsilon_{n}\right] u \\
& =A_{i j l n} \cdot \bar{v}\left[\epsilon_{i}\left(p_{2}-k_{i}+m\right) \epsilon_{j}\left(p_{1}-k_{l}-k_{n}-m\right) \epsilon_{l}\left(p_{1}-k_{n}-m\right) \epsilon_{n}\right] u
\end{aligned}
$$

where $(i, j, l, n)=(1,2,3,4)$ and

$$
A_{i j l n}=\frac{1}{8\left(p_{2} \cdot k_{i}\right)\left(p_{1} \cdot k_{n}\right)\left[\left(p_{1} \cdot k_{l}\right)+\left(p_{1} \cdot k_{n}\right)-\left(k_{l} \cdot k_{n}\right)\right]}
$$

Now, we can use the relations $\left(p_{1}-k_{n}+m\right) \epsilon_{n} u=\epsilon_{n}\left(-p_{1}+k_{n}+m\right) u=\epsilon_{n} k_{n} u\left(\right.$ since $\left.\left(-p_{1}+m\right) u=0\right)$ and $\bar{v} \epsilon_{i}\left(-p_{2}-k_{i}+m\right)=\bar{v}\left(p_{2}+m+k_{i}\right) \epsilon_{i}+\bar{v}\left[-2\left(p_{2} \cdot \epsilon_{i}\right)\right] k_{i}=\bar{v}\left[k_{i} \epsilon_{i}-2\left(p_{2} \cdot \epsilon_{i}\right) k_{i}\right]\left(\right.$ since $\left.\bar{v}\left(p_{2}+m\right)=0\right)$. With the use of these relations one finds

$$
\begin{aligned}
M_{i j l n} & =A_{i j l n} \cdot\left\{-2\left(\epsilon_{i} \cdot p_{2}\right) \cdot \bar{v}\left[\epsilon_{j}\left(p_{1}-k_{l}-k_{n}+m\right) \epsilon_{l} \epsilon_{n} k_{n}\right] u+\bar{v}\left[\epsilon _ { i } k _ { i } \epsilon _ { j } \left(p_{1}-k_{l}\right.\right.\right. \\
& \left.\left.\left.-\quad k_{n}+m\right) \epsilon_{l} \epsilon_{n} k_{n}\right] u\right\}=A_{i j l n} \cdot\left\{2\left(\epsilon_{i} \cdot p_{2}\right) \cdot \bar{v}\left[\epsilon_{j}\left(k_{l}+k_{n}\right) \epsilon_{l} \epsilon_{n} k_{n}\right] u\right. \\
& \left.-\bar{v}\left[\epsilon_{i} k_{i} \epsilon_{j}\left(k_{l}+k_{n}\right) \epsilon_{l} \epsilon_{n} k_{n}\right] u-2\left(\epsilon_{i} \cdot p_{2}\right) \cdot \bar{v}\left[\epsilon_{j} \epsilon_{l} \epsilon_{n}\left(p_{1}+m\right) k_{n}\right] u+\bar{v}\left[\epsilon_{i} k_{i} \epsilon_{j} \epsilon_{l} \epsilon_{n}\left(p_{1}+m\right) k_{n}\right] u\right\}
\end{aligned}
$$

After one additional step of transformations one finds

$$
\begin{aligned}
M_{i j l n} & =A_{i j l n} \cdot\left\{2\left(\epsilon_{i} \cdot p_{2}\right) \cdot \bar{v}\left[\epsilon_{j}\left(k_{l}+k_{n}\right) \epsilon_{l} \epsilon_{n} k_{n}\right] u-\bar{v}\left[\epsilon_{i} k_{i} \epsilon_{j}\left(k_{l}+k_{n}\right) \epsilon_{l} \epsilon_{n} k_{n}\right] u\right. \\
& \left.-4\left(\epsilon_{i} \cdot p_{2}\right) \cdot\left(p_{1} \cdot k_{n}\right) \bar{v}\left[\epsilon_{j} \epsilon_{l} \epsilon_{n}\right] u+2\left(p_{1} \cdot k_{n}\right) \bar{v}\left[\epsilon_{i} k_{i} \epsilon_{j} \epsilon_{l} \epsilon_{n}\right] u\right\}
\end{aligned}
$$

In particular, for the (4321)-amplitude $M_{4321}$ we have

$$
\begin{aligned}
M_{4321} & =A_{4321} \cdot\left\{2\left(\epsilon_{4} \cdot p_{2}\right) \cdot \bar{v}\left[\epsilon_{3}\left(k_{2}+k_{1}\right) \epsilon_{2} \epsilon_{1} k_{1}\right] u-\bar{v}\left[\epsilon_{4} k_{4} \epsilon_{3}\left(k_{2}+k_{1}\right) \epsilon_{2} \epsilon_{1} k_{1}\right] u\right. \\
& \left.-4\left(\epsilon_{4} \cdot p_{2}\right) \cdot\left(p_{1} \cdot k_{1}\right) \bar{v}\left[\epsilon_{3} \epsilon_{2} \epsilon_{1}\right] u+2\left(p_{1} \cdot k_{1}\right) \bar{v}\left[\epsilon_{4} k_{4} \epsilon_{3} \epsilon_{2} \epsilon_{1}\right] u\right\}
\end{aligned}
$$

while

$$
\begin{aligned}
M_{i j l n}^{*} & =A_{i j l n} \cdot\left\{2\left(\epsilon_{i} \cdot p_{2}\right) \cdot \bar{u}\left[k_{n} \epsilon_{n} \epsilon_{l}\left(k_{l}+k_{n}\right) \epsilon_{j}\right] v-\bar{u}\left[k_{n} \epsilon_{n} \epsilon_{l}\left(k_{l}+k_{n}\right) \epsilon_{j} k_{j} \epsilon_{i}\right] v\right. \\
& \left.-4\left(\epsilon_{i} \cdot p_{2}\right) \cdot\left(k_{n} \cdot p_{1}\right) \bar{u}\left[\epsilon_{n} \epsilon_{l} \epsilon_{j}\right] v+2\left(k_{n} \cdot p_{1}\right) \bar{u}\left[\epsilon_{n} \epsilon_{l} \epsilon_{j} k_{i} \epsilon_{i}\right] v\right\}
\end{aligned}
$$


Now, by using the two last formulas one can obtain the explicit expression for all required $M_{i j l n}^{*} M_{4321}$ products. In general, each term of one such a product contains 32 different terms. The total number of terms in the $|M|^{2}$ matrix element is $32 \times 24=768$. However, many of the contributing terms equal zero identically. Analytical computation of the $|M|^{2}$ factor for four-photon case and for arbitrary energies of the colliding particles will be the goal of our next study. These formulas can also be found in [32].

\section{On the creation of macroscopic polyelectrons}

As mentioned above a possibility to create various/higher polyelectrons was discussed by Wheeler [8]. Later this problem has been re-considered by many authors (see, e.g., [11] and references therein). In all these works, however, no actual approaches were proposed which can be used to form polyelectrons. Here we introduce the method in which the radiation-driven ablative implosion is applied to produce linear (or quasi-linear) polyelectrons $e_{n}^{+} e_{m}^{-}$with arbitrary large, in principle, numbers $n$ and $m$. In fact, the proposed approach can be used to obtain even the macroscopic polyelectrons, i.e. the $e_{n}^{+} e_{m}^{-}$systems in which $m \approx N_{A}$ and $n \approx N_{A}$, where $N_{A}$ is the Avogadro number. It is clear that the following annihilation of such polyelectrons will produce an extremely intense flash of annihilation $\gamma$-quanta $E_{\gamma} \approx 0.511 \mathrm{MeV}$. The idea of this two-stage method is simple and transparent. At the first stage some closed spatial area is saturated with the positrons $e^{+}$. The currently used experimental methods allow one to obtain the spatial positron density which is approximately equals $\rho_{0} \approx 1 \cdot 10^{14}$ particle per $\mathrm{cm}^{3}$. At the second stage of the method this low-dense positron gas is rapidly compressed by very intense pulse of the $X$-ray radiation with wavelengths $\lambda \approx 3-10 \AA$. The axial (or cylindrical) symmetry of compression is crucial for workability of this method.

The basic design of a device in which the macroscopic polyelectrons $e_{n}^{+} e_{m}^{-}$can be created must include the two principal parts: (1) a very intense source of $X$-ray radiation (the so-called primary), and (2) a secondary vacuum chamber. The both parts are placed in a cavity with the outer walls which reflect a substantial part of the $X$-rays coming from the overheated primary. Usually, the walls of such cavities (= outer walls, below) are made of heavy metal with large nuclear charge $Z$, e.g., $\mathrm{Pb}(Z=82)$ and/or $\mathrm{Bi}(Z=83)$. The vacuum volume of the secondary chamber is saturated by positrons $e^{+}$to the maximal possible density. Without loss of generality one may represents the primary as a nuclear charge (explosive) covered by the outer shell made from some high- $Z$ metal $(Z \geq 80)$. When this heavy metal is heated from inside to extremely high temperatures $T \geq 50-70 \mathrm{keV}$, then it becomes a very intense source of hard $X$-ray radiation $(\lambda \approx 0.5-5 \AA)$. The flux of $X$-ray radiation from primary reflects from the outer walls of the device and penetrates the walls of the secondary chamber. A very intense flux of the hard $X$-rays also produce photoionization in various electron shells of atoms in the walls of secondary chamber. The emitted photoelectrons $e^{-}$are accelerated to relatively large velocities. In fact, they begin to propagate into the volume of secondary chamber which is saturated with positrons $e^{+}$. Formally, this step corresponds to the formation of the electron-positron mixture (in the secondary chamber) of low density $\approx 10^{16}-10^{18}$ particles per $\mathrm{cm}^{3}$. In the following moments the electron-positron mixture is compressed by the incoming fluxes of $X$-rays of very high intensity. As follows from the general theory of atomic ablation 33 the compression of electron-positron plasma will continue until the radiation pressure from inside of the $\left(e^{-}, e^{+}\right)$-plasma will equalize the ablation pressure of radiation from the hot, heavy element plasma (primary). This simple criterion allows one to evaluate the maximal density of electron-positron plasma at this moment. In fact, as follows from the formula, Eq.(14), derived in [33, the equilibrium density of the $\left(e^{-}, e^{+}\right)$-plasma (in $\left.\mathrm{g} \cdot \mathrm{cm}^{-3}\right)$ will be

$$
\rho_{\left(e^{-}, e^{+}\right)} \approx 0.18 \cdot \rho_{h v} \cdot\left[\frac{M_{\left(e^{-}, e^{+}\right)}}{M_{h v}}\right] \cdot\left(\frac{Z_{e f}}{q}\right)^{2.5} \cdot q \cdot T^{-\frac{1}{2}}
$$

where $\rho_{h v}$ and $M_{h v}$ are the macroscopic density and molar mass of the heavy element plasma, while $\rho_{\left(e^{-}, e^{+}\right)}$and $M_{\left(e^{-}, e^{+}\right)}$are the corresponding density and molar mass of the $\left(e^{-}, e^{+}\right)$-plasma. In Eq. (57) $T$ (in $k e V$ ) is the temperature of the hot, heavy element plasma (e.g., uranium plasma), while the charges $Z_{e f}$ and $q$ are the effective electric charges of the heavy element plasma (at temperature $T$ ) and $\left(e^{-}, e^{+}\right)$-plasma, respectively. At thermal equilibrium the charge $Z_{e f}$ is uniformly related to the temperature $T$ (in $k e V$ ): $Z_{e f}=8.573 \cdot \sqrt{T}$. Now, by assuming that $\rho_{h v}=20 \mathrm{~g} \cdot \mathrm{cm}^{-3}$ and $q=1$ in all cases, one finds that for $T=50 \mathrm{keV}$ the density of electron-positron plasma is $\rho_{\left(e^{-}, e^{+}\right)} \approx 6.640 \cdot 10^{-2} \mathrm{~g} \cdot \mathrm{cm}^{-3}$ (or $\approx 3.645 \cdot 10^{25}$ electron-positron pairs per $\mathrm{cm}^{3}$ ). For $T=70 \mathrm{keV}$ analogous density is $\rho_{\left(e^{-}, e^{+}\right)} \approx 8.545 \cdot 10^{-2} \mathrm{~g} \cdot \mathrm{cm}^{-3}$ (or $\approx 4.690 \cdot 10^{25}$ electron-positron pairs per $\mathrm{cm}^{3}$ ). At such densities the annihilation of $99.999 \%$ of all $\left(e^{-}, e^{+}\right)$-pairs proceeds for a very short time $\tau_{a n n} \leq 25 \Gamma_{2 \gamma}=\frac{50 \hbar}{\alpha^{5} m_{e} c^{2}} \approx 3.11 \cdot 10^{-9}$ sec. Note that the intensity of hard $X$-ray source (primary) vanishes in $\approx 10^{-7}-10^{-6} \mathrm{sec}$. By using the formula for $\tau_{\text {ann }}$ presented above one can easily evaluate the macroscopic annihilation rate and total rate of energy transformation. For any macroscopic amount of the electron-positron mixture $\left(M \geq 1 \cdot 10^{-2}\right.$ gram) these values as well as the total 
$X$-ray brightness are extremely large. It should be mentioned, that in the considered case the mass of extremely hot, heavy element plasma is significantly larger than the original mass of the electron-positron mixture. From here one can expect that the electron-positron plasma will be compressed to densities which are much larger than predicted density $4 \cdot 10^{25}$ electron-positron pairs per $\mathrm{cm}^{3}$. In reality, however, we have to take into account the continuous annihilation of the $\left(e^{-}, e^{+}\right)$-pairs and heating of the compressed electron-positron plasma due to the Compton scattering of annihilation $\gamma$-quanta. Moreover, in any dense plasma the corresponding Fermi limits (see, e.g., [34]) restrict the maximal density of the compressed plasma. Indeed, in any electron-positron mixture there is a minimal pressure

$$
P_{a}=n_{a} T_{e f}\left[1+\frac{\pi^{2}}{15}\left(\frac{T_{a}}{T_{e f}}\right)^{2}+\ldots\right]
$$

which is consistent with the Fermi degeneracy of the electrons/positrons. In Eq.(58) the subscript $a$ equals + for positrons and - for electrons, $T_{a}$ is the actual temperature, while $T_{e f}=\frac{(9)^{\frac{1}{3}}}{5}\left(\frac{\pi^{2} \hbar^{2}}{m_{e}}\right) \rho_{b}^{\frac{2}{3}}$ is the so-called 'equivalent Fermi temperature' of the $\left(e^{-}, e^{+}\right)$-mixture and $\rho_{b}=\max \left(\rho_{+}, \rho_{-}\right)$is the density of the main component (i.e. electron and/or positron component). For almost equimolar $\left(e^{-}, e^{+}\right)$-mixtures the total pressure $P=P_{+}+P_{-} \approx 2 P_{-}$. As follows from Eq.(58) at $T_{a} \approx T_{e f}$ the actual pressure in the electron-positron mixture can be significantly larger (by a few orders of magnitude) than the pressure determined from the usual formula $P_{a}=n_{a} T_{a}$.

Note that the macroscopic polyelectrons, and even linear polyelectrons, show a number properties which cannot be found in any other macroscopic system. Indeed, all regular atomic and molecular systems in our world are the Born-Oppenheimer systems. In such systems all usual positive particles are heavy, while all negatively charged particles (electrons) have significantly (in $\approx 2000$ times) smaller mass. A few recently created systems consisting of anti-particles are also the Born-Oppenheimer systems, since the masses of negative particles in such systems are much larger than the masses of positive particles. In contrast with this, the electron-positron plasma is an example of the system from different, non-Born-Oppenheimer world.

\section{Conclusion}

Thus, we have considered the annihilation of electron-positron pairs in some polyelectrons, including the $\mathrm{Ps}^{-}$ion, bipositronium $\mathrm{Ps}_{2}$ system and $e_{2}^{+} e_{3}^{-}$ion. Annihilation of the electron-positron pairs in these polyelectrons is considered in detail. A number of problems related to the annihilation of the $\left(e^{-}, e^{+}\right)$-pairs discussed in this study have never been considered. In particular, we derive the analytical expression for the amplitude-square $|M|^{2}$ of the threephoton annihilation of $\left(e^{-}, e^{+}\right)$-pair at arbitrary energies of the colliding particles. Analogous expression for the $|M|^{2}$ factor in the case of four-photon annihilation will be produced in our next work. The method which can be used to produce macroscopic polyelectrons $e_{n}^{+} e_{m}^{-}$in an external radiation field is briefly discussed. This approach is based on the idea of atomic compression.

\section{Acknowledgements}

It is a pleasure to thank D.G.C. (Gerry) McKeon for helpful discussions.

\section{References}

[1] R.J. Drachman, Can. J. Phys. 60, 494 (1982).

[2] R.K. Wehner, Solid State Comm. 7, 457 (1969).

[3] R.R. Sharma, Phys. Rev. 170, 770 (1968).

[4] D.M. Schrader, Phys. Rev. Lett. 92, 043401 (2004).

[5] G. Bao and T.Y. Shi, Phys. Rev. A 67, 042505 (2003).

[6] J. Paul, Nucl. Instr. and Meth. Phys. Res. B 221, 215 (2004).

[7] A.E. Ruark, Phys. Rev. 68, 278 (1945).

[8] J.A. Wheeler, Ann. N.Y. Acad. Sci. 48, 219 (1946). 
[9] E.A. Hylleraas, Phys. Rev. 71, 491 (1947).

[10] E.A. Hylleraas and A. Ore, Phys. Rev. 71, 913 (1947).

[11] D.M. Schrader, in: Positron Annihilation, Eds. P.G. Coleman, S.C. Sharma, and L.M. Diana, (North-Holland, Amsterdam, 1982) p.71.

[12] S.I. Kryuchkov, J. Phys. B 27, L61 (1994).

[13] A.M. Frolov, Phys. Rev. A 57, 2436 (1998).

[14] The NIST Reference on Constants, Units and Uncertainty, see: http://physics.nist.gov/cuu/Constants/index.html

[15] A.M. Frolov, J. Phys. A 40, 6175 (2007).

[16] A.I. Akhiezer and V.B. Berestetskii, Quantum Electrodynamics, (4th revised ed., Nauka, Moscow, (1981), in Russian), $\S 4.5$ and $\S 5.5$.

[17] I. Harris and L.M. Brown, Phys. Rev. 105, 1656 (1957).

[18] A.K. Bhatia and R.J. Drachman, Phys. Rev. A 28, 2523 (1983).

[19] Y.K. Ho, Phys. Lett. A 144, 237 (1990).

[20] A.P. Mills, Jr., Phys. Rev. Lett. 50, 671 (1983).

[21] D.H. Bailey and A.M. Frolov, Phys. Rev. A 72, 014501 (2005).

[22] A.M. Frolov, S.I. Kryuchkov and V.H. Smith, Jr., Phys. Rev A 51, 4514 (1995).

[23] S. Bubin and L. Adamowicz, Phys. Rev A 74, 052502 (2006).

[24] P.A.M. Dirac, Proc. Cambr. Phil. Soc. 26, 361 (1930).

[25] F. Mandl and G. Shaw, Quantum Field Theory, (J. Wiley and Sons, New York (1984)), §6.1.

[26] E.M. Lifshitz, DAN SSSR 60, 211 (1948).

[27] D. Ivanenko and A. Sokolov, DAN SSSR 61, 51 (1948).

[28] A. Ore and J.L. Powel, Phys. Rev. 75, 1696 (1949).

[29] J.D. Bjorken and S.D. Drell, Relativistic Quantum Mechanics, (McGraw-Hill Book Company, New York (1964)), Sec. 7.

[30] W. Greiner and J. Reinhardt, Quantum Electrodynamics, (Springer, Berlin, 3rd Ed., (2003)), p. 213.

[31] K. Melnikov and A. Yelkhovski, Phys. Rev D 62, 116003 (2000).

[32] see: http://www.apmaths.uwo.ca/ afrolov/3phtr.pdf

[33] A.M. Frolov, Can. J. Phys. 84, 823 (2006).

[34] G.S. Fraley, E.J. Linneburg, R.J. Mason and R.L. Morse, Phys. of Fluids 17, 474 (1974). 


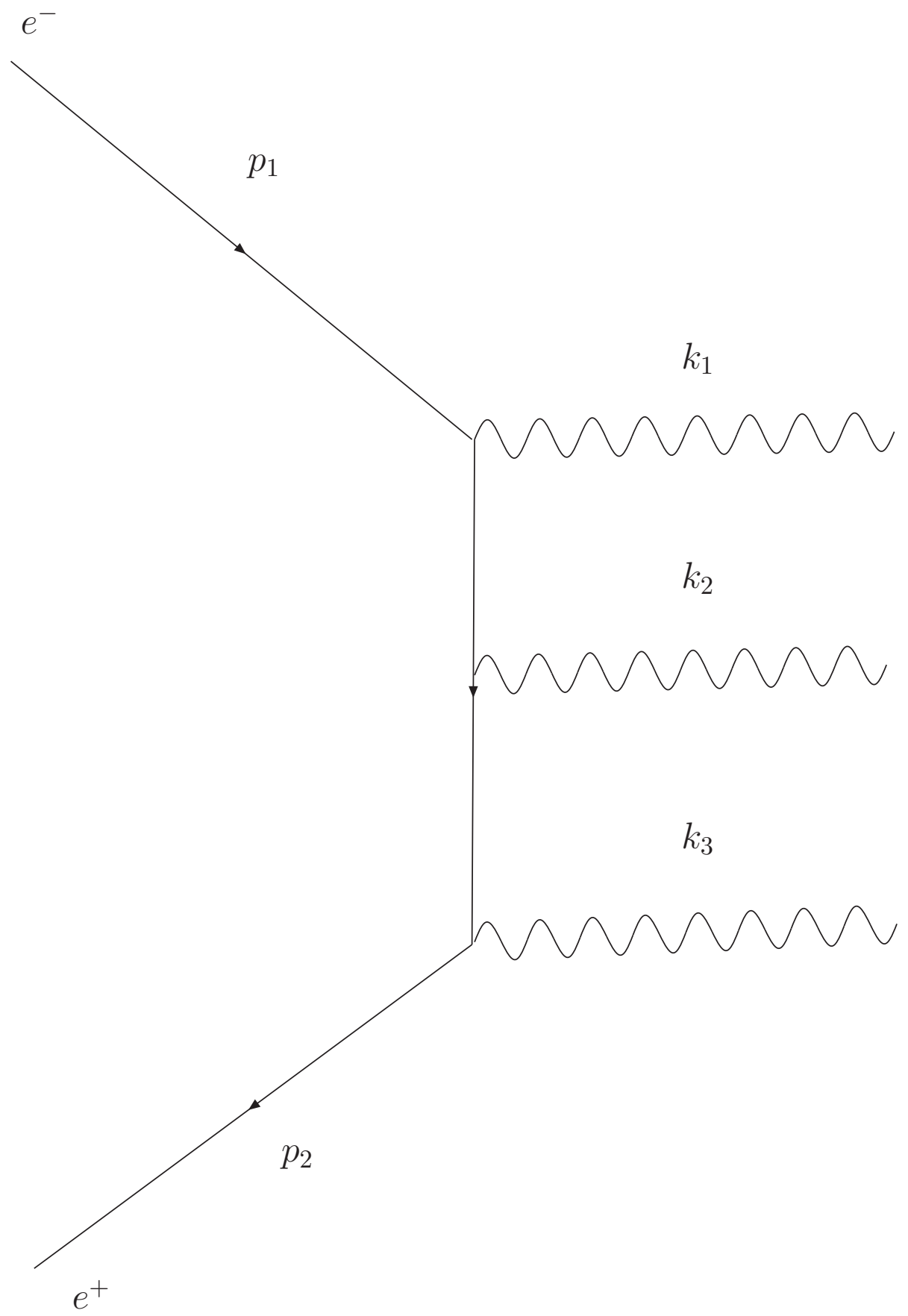

Figure 1: Graph of $\left(e^{-}, e^{+}\right)$-pair annihilation into three photons. The (1,2,3)-graph is shown. 


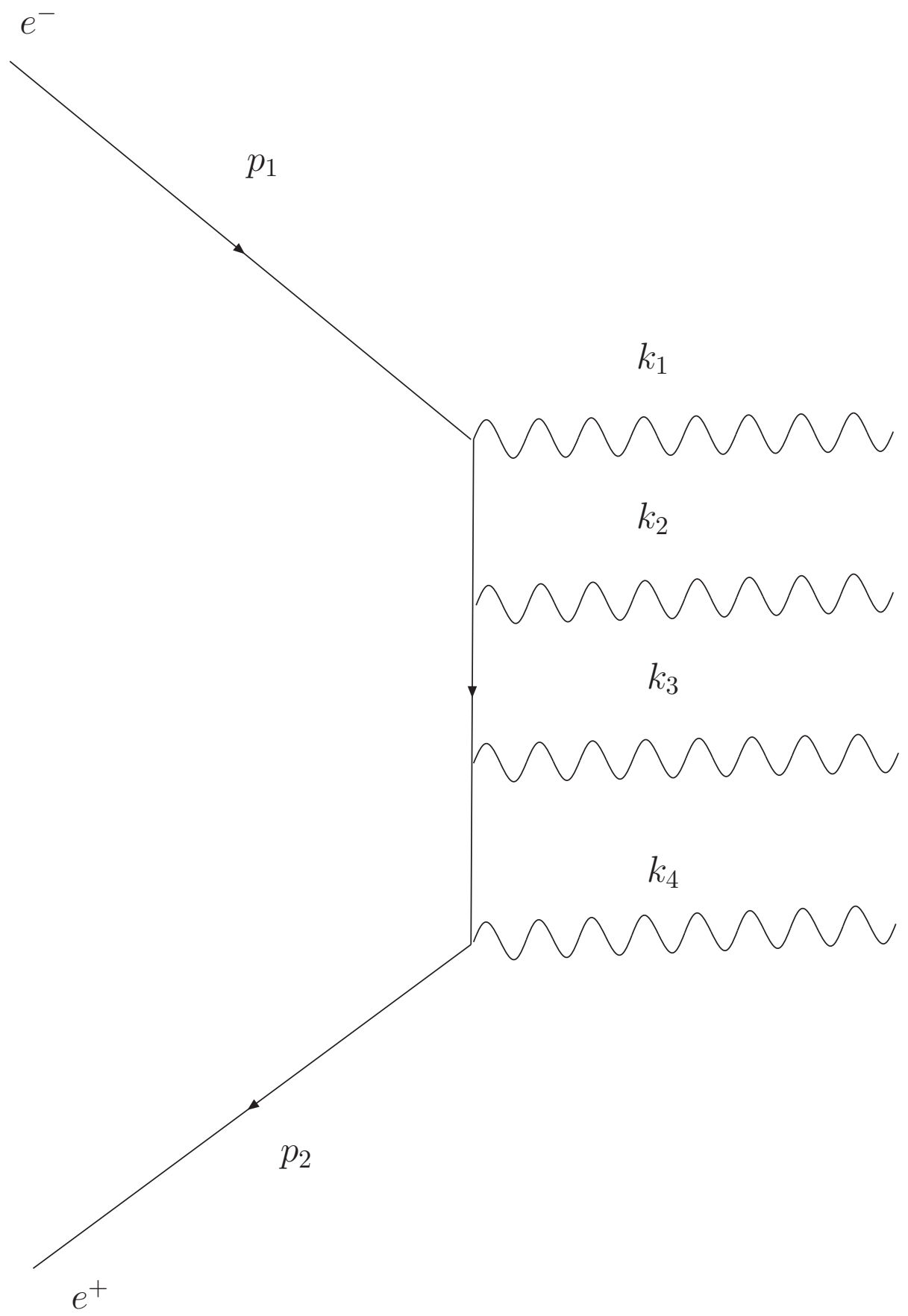

Figure 2: Graph of $\left(e^{-}, e^{+}\right)$-pair annihilation into four photons. The $(1,2,3,4)$-graph is shown. 